\title{
Synchronizing an oscillatory medium: The speed of pacemaker-generated waves
}

\author{
Jan Rombouts $\odot^{*}$ and Lendert Gelens $\odot^{\dagger}$ \\ Laboratory of Dynamics in Biological Systems, Department of Cellular and Molecular Medicine, \\ University of Leuven (KU Leuven), B-3000 Leuven, Belgium
}

(Received 25 February 2020; accepted 21 September 2020; published 7 October 2020; corrected 9 November 2020 and 26 April 2021)

\begin{abstract}
In an oscillatory medium, a region which oscillates faster than its surroundings can act as a source of outgoing waves. Such pacemaker-generated waves can synchronize the whole medium and are present in many physical and biological systems, where they are a means of transmitting information. Through numerical simulations, we quantify how the properties of the pacemaker and the underlying limit cycle determine the wave speed, as well as the speed with which they overtake the medium. We compare oscillators based on two of the main mechanisms that generate oscillations in biochemical systems: bistability and time delay. We show that these mechanisms produce oscillations whose wave propagation properties differ markedly. While both types of oscillatory media admit waves that propagate linearly outwards, the dependence of the wave speed on a timescale separation parameter is different between the two. If timescale separation is lost, waves no longer spread linearly. Finally, we quantify the effects of pacemaker size, the frequency difference with the surroundings, and the diffusion strength.
\end{abstract}

DOI: 10.1103/PhysRevResearch.2.043038

\section{INTRODUCTION}

Nature is full of periodic phenomena. In biology, familiar examples include the ubiquitous 24-h circadian rhythms, the cell division cycle, and heart contractions [1]. In those systems, periodicity is essential for survival and development. Many of these rhythms are generated by biochemical mechanisms, in which proteins and genes interact through multiple feedback loops to generate cycles in protein concentration and activity.

Understanding the properties of such biochemical oscillators has been an important driver for the development of mathematical models. These models themselves exist on a range of scales, with on the one hand detailed descriptions of all biochemical interactions and on the other hand more abstract representations, meant to understand generic features of these oscillators. Examples of the latter include the phasedynamics approaches pioneered by Winfree [2] and Kuramoto [3], which have been highly influential in understanding how different oscillators influence each other. An entirely different type of theory was developed by Thomas [4], who formalized the study of feedback loops [5]. Thomas' rules state that positive feedback and negative feedback are essential to achieve multistability and oscillations, respectively. For a system to cycle, there needs to be a mechanism that can reset it, which is provided by the negative feedback. However, the presence

\footnotetext{
*jan.rombouts@kuleuven.be

†lendert.gelens@kuleuven.be

Published by the American Physical Society under the terms of the Creative Commons Attribution 4.0 International license. Further distribution of this work must maintain attribution to the author(s) and the published article's title, journal citation, and DOI.
}

of negative feedback is not enough to guarantee oscillations. The two main mechanisms which complement negative feedback are an additional positive feedback and/or a time delay [Figs. 1(a) and 1(b)]. Whereas a sufficiently delayed negative feedback can lead the system to oscillate, robustness of the amplitude of the oscilllations is often achieved when positive feedback loops are added to the system [6]. These positive feedback loops can lead to multiple steady states, which govern the dynamics of the system. Oscillations consist of slow progress along branches of steady states, with quick jumps between them. Such oscillations are called relaxation oscillations and play an important role in many natural phenomena. Excellent explanations of how different kinds of motifs and feedback loops can give rise to oscillations can be found in the papers by Novák and Tyson [7] and Ferrell et al. [8].

Typically, biochemical systems are modeled using ordinary differential equations (ODEs), with one equation for each concentration or activity involved. The evolution of the system is generated by chemical reactions. In such systems, a time delay is usually implicit, for example, through intermediate variables. Another way to model time delays is by introducing them explicitly in a delay differential equation (DDE), where the current rate of change of the system may depend on the past state. When the current rate of change depends on the whole history, and not on a specific past point in time, delays can also be modeled using integrodifferential equations. Time delays can be introduced to capture a travel or signaling time, or a number of - possibly unknown-intermediate processes. Even though they are a crude approximation of biological reality, simple time-delay equations have successfully captured key features of a number of oscillatory biological systems. The book by Erneux [9] contains plenty of examples.

At first sight, the cycles of an oscillator governed by underlying bistability may not be very different from an oscillator where a highly nonlinear, time-delayed negative feedback is 
(a)

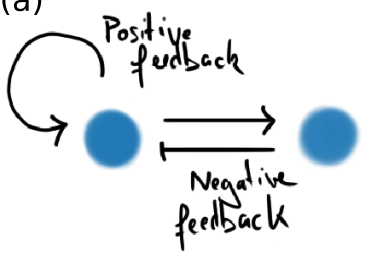

(c)

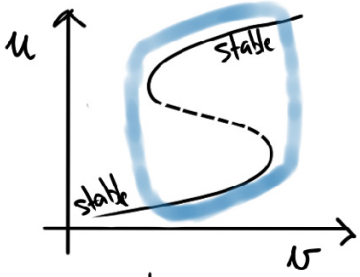

(b)

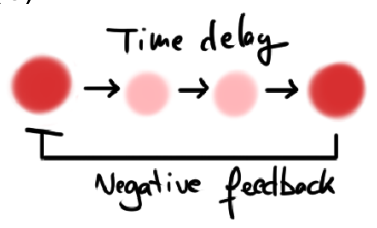

(d)

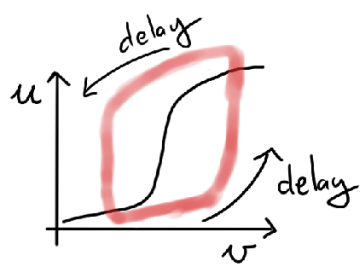

(e)

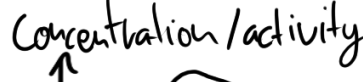

(f)
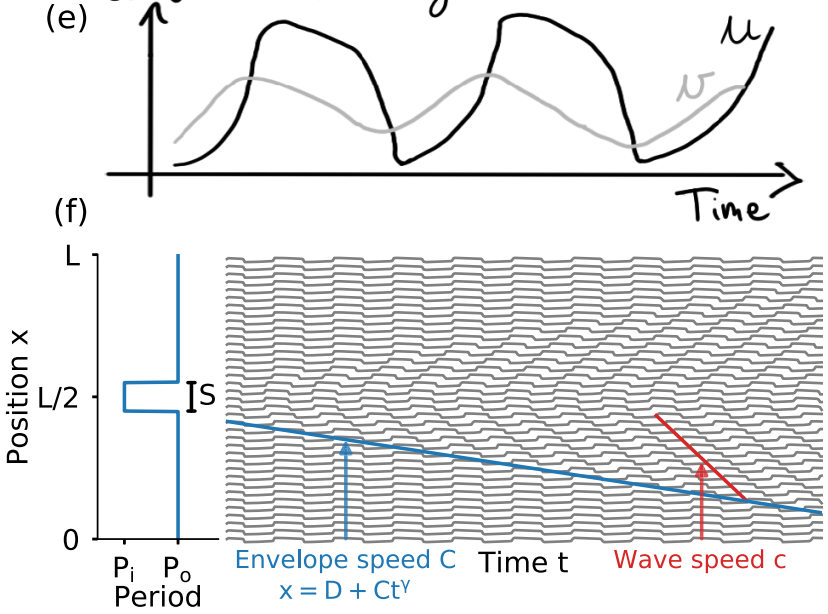

FIG. 1. Oscillations in time and space. (a), (c) A negative feedback combined with a positive feedback can lead to oscillations based on jumping between different branches of a bistable system. (b), (d) Time-delayed negative feedback is another mechanism which may lead to large-amplitude biochemical oscillations. (e) Time series which could correspond to either of the two motifs. (f) When coupled in space, oscillations can give rise to traveling waves. Such waves are often sent out by an inhomogeneity in the system, called the pacemaker. Two different speeds are of interest: the speed of the wave itself, and the speed by which the wave permeates the surrounding medium, which we will call the envelope speed.

generating the oscillation. The time series for such systems may be very similar [see Figs. 1(a)-1(e) and Figs. 2(a)-2(f)]. In this paper, however, we show that these systems differ markedly when coupled in space, and that the main difference lies in how they generate waves.

A natural place to find biochemical oscillations is inside a cell, a heterogeneous environment which, especially for large cells such as egg cells, is not well mixed. A spatial description is thus necessary to capture the dynamics in the cell. Concentrations and activities can now vary in time and space, and ODEs give way to partial differential equations (PDEs) that include diffusion of chemicals.

Oscillatory systems which are coupled in space show a wealth of patterns, either stationary, chaotic, or in the form of traveling waves [10]. In cell biology, traveling waves usually
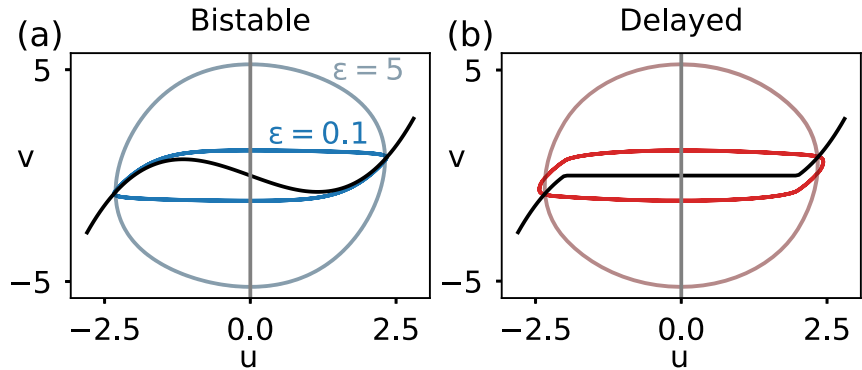

(c)

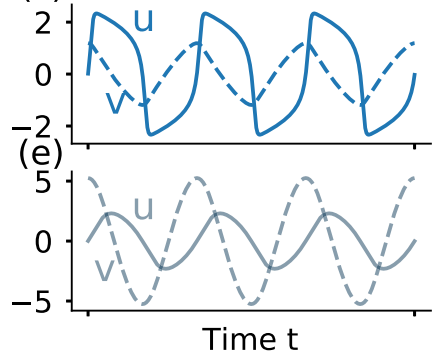

(g)

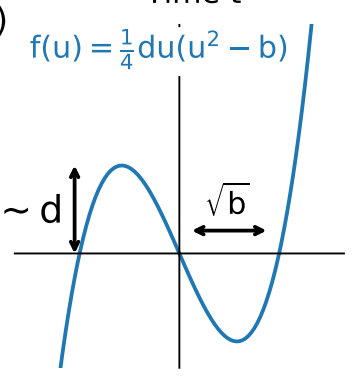

(d)

(f)
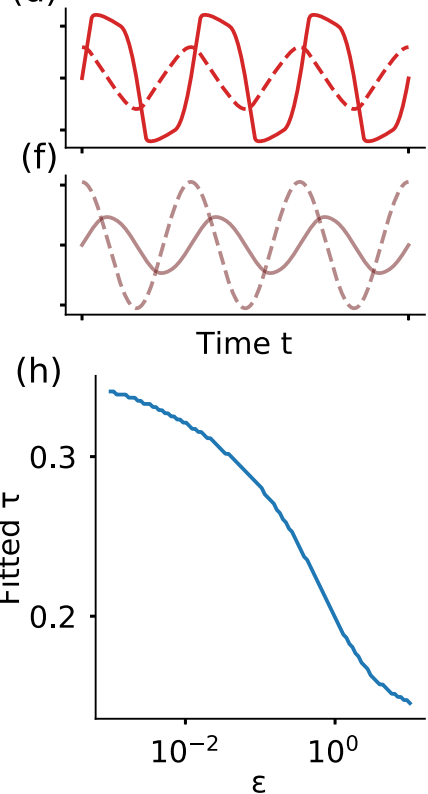

FIG. 2. (a), (b) Phase-plane depiction of the limit cycle in the bistable oscillator model (a) and the delayed oscillator model (b) for two different values of $\varepsilon$. (c)-(f) Time series of the bistable (c), (e) and delayed (d), (f) oscillator for $\varepsilon=0.1$, relaxationlike oscillations (c), (d) and $\varepsilon=5$, sinusoidal oscillations (d), (f). Time has been rescaled such that the period is equal to 10 . In panels (c)-(f), the $u$ variable is represented by the solid line and the $v$ variable by a dashed line. $(\mathrm{g})$ The cubic function $f$ is modified by the parameters $d$ and $b$, which can however be absorbed in the other parameters of the model. (h) In order to align the delayed oscillator with the bistable oscillator, we choose $\tau$ as a function of $\varepsilon$ such that the amplitude in the $v$ direction of both oscillators is as close as possible.

function to transmit information over long distances [11]. Examples include mitotic waves in the cell cycle of Xenopus frog embryos [12] and Drosophila fly embryos [13], actin waves which play a role in cell migration $[14,15]$, and many others $[1,16]$. Besides waves in which a protein concentration or enzymatic activity is propagated, there are many wave phenomena of an electrical nature, in neurons or cardiac cells $[17,18]$. Here it is a voltage, instead of a chemical concentration, which is transmitted in space.

Traveling waves often originate at inhomogeneities in the system. An inhomogeneity where the frequency of the oscillations is higher than in the surrounding medium can send out waves and entrain the rest of the medium [Fig. 1(f)]. Such a region is therefore called a pacemaker. In two-dimensional systems, pacemakers sending out waves generate target patterns. Some of the clearest examples of such patterns come 
TABLE I. Estimates of wave speeds in various chemical and biological systems.

\begin{tabular}{lcc}
\hline \hline System & Wave speed & Ref. \\
\hline Actin waves (various cells) & $0.01-0.15 \mu \mathrm{m} / \mathrm{s}$ & {$[15]$} \\
Mitosis in early embryos & $0.5-1 \mu \mathrm{m} / \mathrm{s}$ & {$[12,13,26]$} \\
Oxidation of CO on Pt(110) reaction & $1-4 \mu \mathrm{m} / \mathrm{s}$ & {$[20,21]$} \\
cAMP signaling & $5-7 \mu \mathrm{m} / \mathrm{s}$ & {$[28]$} \\
Yeast glycolysis & $10-100 \mu \mathrm{m} / \mathrm{s}$ & {$[24]$} \\
Belousov-Zhabotinsky reaction & $50-100 \mu \mathrm{m} / \mathrm{s}$ & {$[19,29]$} \\
Action potential in cardiac tissue & $50-70 \mathrm{~cm} / \mathrm{s}$ & {$[18,30]$} \\
Action potential in squid giant axon & $20 \mathrm{~m} / \mathrm{s}$ & {$[31]$} \\
\hline \hline
\end{tabular}

from chemistry and include a dish filled with reactants for the Belousov-Zhabotinsky reaction [19] or the oxidation of CO on $\mathrm{Pt}(110)$ [20,21]. In such chemical systems, pacemakers can appear through impurities such as dust particles, or they can be induced, for example, to suppress chaotic behavior [22]. In biological systems, target patterns occur in cyclic adenosine monophosphate (cAMP) signaling in Dictyostelium discoideum [23], cardiac tissue [18], yeast glycolysis [24], neural tissue [25], and cell-free extracts of Xenopus laevis frog eggs [12], where nuclei act as pacemakers to organize the dynamics $[26,27]$.

Pacemaker-generated waves have a clear function in these biological systems: they synchronize the system over long distances and transmit information inside the cell or between cells $[1,11,16]$. To ensure proper coordination of these biological processes, it is essential that the waves spread sufficiently quickly through the medium (see Table I).

Some of the examples we mentioned above concern excitable media, instead of oscillatory media. However, the dynamics of an excitable system are often guided by the existence of multiple stable branches along which the system proceeds, just as oscillators based on bistability. In such systems, excitability and oscillations may be present in the same system in different, but close, parameter regimes. The waves in such systems, especially for large timescale separation, are related and can be studied using the propagation of fronts [32]. Different types of traveling fronts exist and play a role in biology [33-35]. In this paper, however, we always consider oscillatory media.

Wave phenomena and pacemaker dynamics have been studied mathematically for a long time, mainly using approximations based on phase-reduction methods [3,36,37] or singular perturbation methods [32]. These methods provide essential qualitative understanding of when pacemakers send out waves. However, quantitative studies of what properties of the pacemakers and of the limit cycle itself determine the speed of propagation are rare. Most of these studies have focused on analytically tractable models, such as $\lambda-\omega[38]$ or Stuart-Landau oscillators, and the complex Ginzburg-Landau equation (CGLE) [39]. For example, for the CGLE in two dimensions the influence of the size of the pacemaker on the wave number of resulting target patterns has been studied for disk- and square-shaped pacemakers [40] and ring-shaped pacemakers [41]. A study of pacemakers in the CGLE in one dimension can be found in Ref. [42].
In this paper, we numerically study oscillatory media with a single pacemaker in the middle of the domain. Our goal is to quantify, first, the speed of waves sent out by this pacemaker and, second, the speed by which such waves overtake the whole medium. We call the latter the envelope speed [Fig. 1(f)]. The structure of the paper is as follows. In Sec. II, we introduce the two sets of equations we use for oscillators based on bistability and time delay, respectively. We detail the algorithm we use for the detection of the speed in Sec. III, and we discuss the results of our simulations in Sec. IV. First, we explain the influence of the shape and parameters of the limit cycles themselves, with a main focus on the timescale separation (Sec. IV B). After showing that these results are not specific to our equations, but also appear in existing cell cycle models (Sec. IV C), we then discuss how pacemaker size and period influence wave speed (Sec. IV D). Finally, we show how the diffusion strength influences the waves in Sec. IV E.

\section{OSCILLATORS BUILT ON BISTABILITY AND ON TIME DELAY}

\section{A. The bistable oscillator}

The equations we use for the oscillator based on bistability, here referred to as the bistable oscillator, read

$$
\begin{aligned}
u_{t} & =\varepsilon^{-1}\left(v-\frac{1}{4} d u\left(u^{2}-b\right)\right)+D_{u} u_{x x}, \\
v_{t} & =a-u+D_{v} v_{x x} .
\end{aligned}
$$

This set of equations is a variant of the Van der Pol oscillator [43] and the related FitzHugh-Nagumo equations [44,45]. We call it bistable not because these equations themselves admit multiple stable solutions, but because for a fixed value of $v$, the $u$ variable can evolve towards two stable states, the branches of the cubic function. In terms of motifs, this is due to a positive feedback of $u$ on itself. When $v$ is allowed to evolve in time, the system can be excitable or oscillatory, depending on the value of $a$. Here we only consider values of $a$ where the system oscillates. In such oscillations, the system slowly progresses along the branches of the cubic function, with quick jumps between them. This happens for low values of $\varepsilon$. For increasing $\varepsilon$, the system transitions from relaxation-type oscillations to more harmonic oscillations with sinusoidal waveforms for $u$ and $v$ [Figs. 2(a), 2(c), and 2(e)].

The shape of the cubic function can be modified by the parameters $d$ and $b$ [Fig. 2(g)]. However, using a suitable scaling of the variables, these parameters can be absorbed into $\varepsilon$ and $a$ (see Appendix A). From now on we take $d=1$ and $b=4$.

Note that we allow diffusion in both the fast and the slow variable, which is unlike applications in which these equations are used for modeling action potentials. In those systems, there is typically only diffusion in the fast variable $u$. Here, we regard $u$ and $v$ as generic variables that can stand for any kind of chemical concentration; therefore we allow both of them to diffuse.

\section{B. The delayed oscillator}

We compare this system to one without bistability, where the two branches of the nullcline are replaced by a time delay. 
This delayed oscillator is described by

$$
\begin{aligned}
u_{t} & =\varepsilon^{-1}(v(t-\tau)-\tilde{f}(u))+D_{u} u_{x x}, \\
v_{t} & =a-u+D_{v} v_{x x}, \\
\tilde{f}(u) & =f(u) \kappa_{[-2,2]}(u),
\end{aligned}
$$

where $\kappa_{[-2,2]}(u)=0$ if $-2<u<2$ and 1 otherwise. The function $\tilde{f}$ was chosen to overlap with the cubic function $f(u)=\frac{1}{4} u\left(u^{2}-4\right)$, used in the bistable oscillator, far from the origin [Fig. 2(b)]. The delay $\tau$ is chosen such that the amplitude of the $v$ variable in the delayed system is equal to the $v$ amplitude in the bistable system with the same $\varepsilon$, with typical values around 0.3 [Fig. 2(h)]. Through this choice of the function $\tilde{f}$ and the time delay $\tau$, we assure that the time-delay system is as close as possible to the bistable oscillator if one would inspect the time series or the phase plane [compare Figs. 2(c) and 2(d) and Figs. 2(e) and 2(f). We have effectively replaced the progression along the branches in the bistable system by an overshoot due to delay in the delayed system. From a mathematical point of view, both systems are not trivial to compare directly since there are significant mathematical differences between ODEs and DDEs. However, we think it is useful to make this comparison, since in real biological or chemical systems, it is often not clear what the underlying dynamical system is by mere inspection of the time series. The comparison is thus more conceptual than technical.

Even though the choice of $\tilde{f}$ is artificial, this delayed oscillator is an example of a delayed negative feedback loop such as, for example, the Goodwin oscillator [46] or the delayed cell cycle oscillator [47]. From a different viewpoint, this equation models the variable $v$ which brings about its own decline in a time-delayed, highly nonlinear way. For $\varepsilon \rightarrow 0$, we obtain from the first equation

$$
v(t-\tau) \approx \tilde{f}(u) \Rightarrow u=\tilde{f}^{-1}[v(t-\tau)]=g[v(t-\tau)],
$$

where the function $g$ is the inverse of $\tilde{f}$, a steplike response function. For very small $\varepsilon$, the system is approximately given by

$$
v^{\prime}=a-g[v(t-\tau)],
$$

which is a negative feedback system: $v$ starts out small, such that $g[v(t-\tau)]$ is negative. This means $a-g[v(t-\tau)]$ is positive, leading to an increase in $v$. At a time $\tau$ after $v$ crosses zero, $g[v(t-\tau)]$ becomes positive and $v$ starts decreasing, provided $a$ lies in $(-2,2)$.

The time series of the delayed oscillator similarly show a transition from relaxationlike oscillations to more sinusoidal oscillations for increasing values of $\varepsilon$ [Figs. 2(b), 2(d), and 2(f)].

\section{NUMERICAL METHODS AND ALGORITHM FOR SPEED DETECTION}

Our system consists of a one-dimensional domain of length $L$ with periodic boundary conditions. We take $L=400$ as standard domain length. In the middle of the domain we define a pacemaker region of size $S$ which oscillates faster than the surroundings [Fig. 1(f)]. In order to compare wave speeds in different systems and with different parameters, we
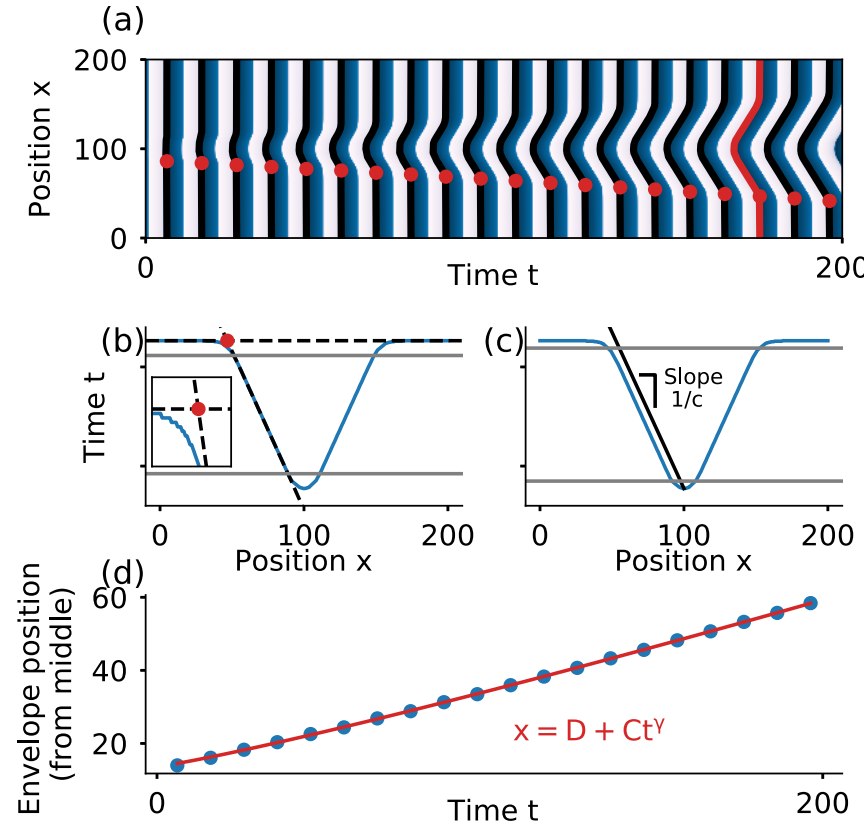

FIG. 3. Detection of the envelope exponent, envelope speed, and wave speed. (a) Space-time plot of the $u$ variable. Black lines are the profiles where $u$ crosses 0 from below. The red profile is the one used in the other panels. The red dots denote the detected envelope location. (b) Envelope detection from a profile. (c) Wave speed detection. (d) Envelope points as a function of time together with fit, from which the envelope speed can be obtained.

always rescale time such that the period of the uniform spatial oscillation is $P_{o}$. In the pacemaker region, we rescale time such that the period there is $P_{i}<P_{o}$. Standard values are $P_{o}=10$ and $P_{i}=9.5$. Although a pacemaker region can be induced by any space-dependent parameter, here we use time rescaling to ensure that only the frequency, and not the shape of the limit cycle, is altered in the pacemaker region.

We perform our numerical simulations using a pseudospectral algorithm for the bistable system and an implicit algorithm for the delayed oscillator (see Appendix B for more details). These algorithms were chosen because they provide a good compromise between accuracy, simplicity, and computational cost.

After simulating the system for a total time of $T=1000$ (this corresponds to 100 periods for our default of $P_{o}=10$ ), we detect whether a wave propagates linearly outward from the pacemaker and, if so, what the wave speed and envelope speed are. Figure 3 shows the details of the algorithm we use. First, we iterate over each spatial position $x_{i}$ to detect all the points $\left(x_{i}, t_{i, j}\right)$ for which the $u$ variable crosses zero from below. For the first position $x_{0}$, each of the points $\left(x_{0}, t_{0, j}\right)$ will be the first point of a profile. For each of the points $\left(x_{i}, t_{i, j}\right)$ at subsequent spatial locations, we find the closest point for the previous $x$ value $\left(x_{i-1}, t_{i-1, j^{\prime}}\right)$. We append the point $\left(x_{i}, t_{i, j}\right)$ to the profile containing this previous point. In this way we obtain profiles separated in time [Fig. 3(a), black lines]. For such a profile, we can plot the $t$ coordinates as functions of the $x$ coordinates. This has a "V" shape with two horizontal extensions [Figs. 3(b) and 3(c)]. We define the envelope position as the transition point from the horizontal 
part to the $\mathrm{V}$ part. Since this transition is smooth, we use the following method to detect this point: first, we smooth the profile using a seven-point moving average. Next, we fit a function of the form $t=a+b t^{\beta}$ through the middle part of the $\mathrm{V}$ branch [between the gray horizontal lines in Fig. 3(b)]. The envelope position is the intersection of the horizontal line through the leftmost point of the profile and this fitted function [dashed lines in Fig. 3(b)]. If there is an outgoing wave, its speed can be detected from such a profile as well [Fig. 3(c)]. For this, we fit a straight line through the middle of a branch of the V. The wave speed is the inverse of the slope of this line.

Once the envelope points have been detected for all profiles [Fig. 3(a), red dots], we omit the first point and the points whose distance to the boundary is less than $L / 20$, and next we fit a function of the form $x=D+C t^{\gamma}$ to the outgoing envelope [Fig. 3(d)]. For $\gamma \approx 1, C$ corresponds to the envelope speed (see also Fig. 1). The periodic boundary conditions do not influence the results, which we tested by trying different domain lengths $L$.

\section{RESULTS}

\section{A. Asymmetry weakly influences the wave speed}

First, we look at the influence of the parameter $a$. For $a=0$, oscillations in the bistable model are symmetric and spend equal amounts of time on the left and right branches of the cubic nullcline [Fig. 4(c)]. For the delayed model, the oscillations are also symmetric for $a=0$ [Fig. 4(d)]. If $a>0$, the system spends a longer time on the right branch of the nullcline [Fig. 4(e)]. The shape of the limit cycle in the phase plane remains largely unchanged in the bistable model [Fig. 4(a)]. However, in the delayed oscillator, the limit cycle is shifted to higher values of $v$ [Figs. 4(b) and 4(f)]. Oscillations cease to exist if $|a|$ is too large.

Asymmetric oscillations generate waves with a slightly larger speed in both models, although the effect is small [Fig. 4(g)]. Due to symmetry, the wave speed for $-a$ is the same as that for $a$. In the following, we take $a=0$ for definiteness.

\section{B. Influence of timescale separation}

The timescale separation parameter $\varepsilon$ determines the character of the oscillations (Figs. 2 and 5(a)-5(c)]. In both the bistable model and the delayed model, low values of $\varepsilon$ correspond to oscillations with quick jumps between two branches. Higher values of $\varepsilon$, on the other hand, correspond to harmonic oscillations where both $u$ and $v$ show sinusoidal time series.

When timescales are well separated (small $\varepsilon$ ), waves spread linearly or even slightly faster [Figs. 5(d) and 5(g)]. When $\varepsilon$ is increased, the wave gradually spreads in a more diffusionlike way ( $\gamma$ decreases) [Figs. 5(e) and 5(h)]. For even higher values of $\varepsilon$, phase slips occur [Figs. 5(f) and 5(i)]. These same three regions of qualitatively different behavior are found for both types of oscillator [Fig. 5(j)]. This shows that, from the mere observation that waves are present, we cannot distinguish between the two mechanisms. A different story holds, however, when we examine the speed of these waves.
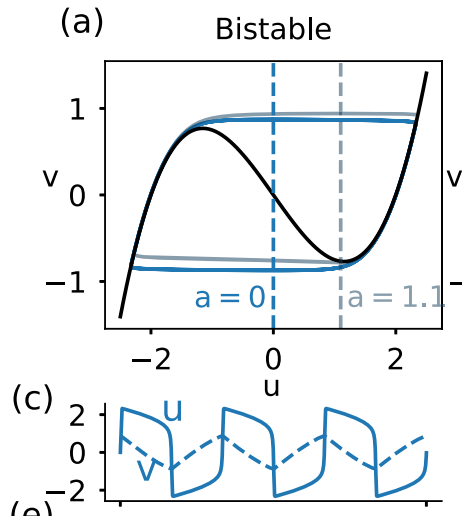

(e)

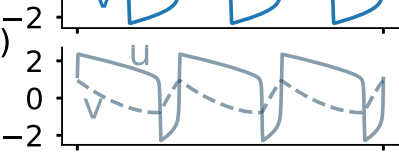

(g)

(b) Delayed
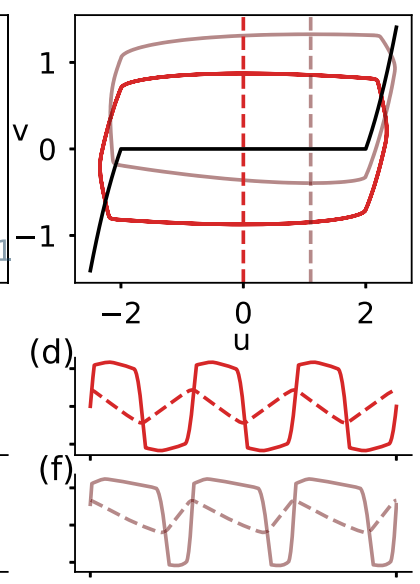

Time $\mathrm{t}$

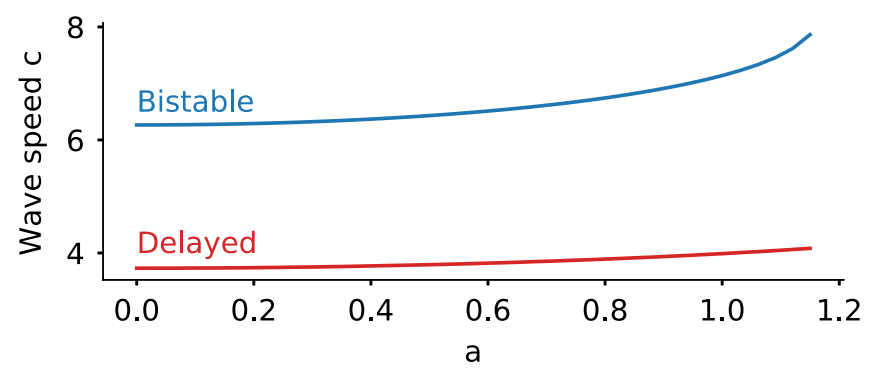

FIG. 4. The parameter $a$ determines the symmetry of the oscillations, but has little effect on the wave speed. (a), (b) Limit cycle for $a=0$ and $a=1.1$ in the bistable oscillator (a) and the delayed oscillator (b). (c)-(f) Time series corresponding to the limit cycles shown in panels (a) and (b). For panels (c) and (d): $a=0$ (symmetric waveform). For panels (e) and (f): $a=1.1$. (g) Wave speed as a function of $a$ for both models. In all plots, $\varepsilon=0.01$.

In the region where $\gamma>0.9$, we compute the speed of the outgoing wave $c$ [Fig. 5(k)]. Interestingly, while we set up the systems in such a way that both bistable and delayed systems have very similar waveforms [Figs. 2 and 5(a)-5(c)], the dependence of wave speed on $\varepsilon$ differs significantly. Increasing $\varepsilon$ lowers the wave speed for the bistable oscillator, whereas in the delayed system it is nearly independent of $\varepsilon$. For the bistable model, there are two regimes for the wave speed, with a transition around $\varepsilon=0.01$. Note that for higher values of $\varepsilon$, when both oscillators are close to harmonic, their wave speed is nearly equal.

The envelope speed $C$ follows the trend of the wave speed [Fig. 5(1)]. If we know the wave speed, we can determine the envelope speed using the formula

$$
C=\frac{P_{o}-P_{t}}{P_{o}} c .
$$

This formula can be derived from the following geometrical argument. Referring to the notation in Fig. 5(m), if $\left(t_{1}, x_{1}\right)$ is the first envelope point and $\left(t_{2}, x_{2}\right)$ is the second, we have $t_{2}-t_{1}=P_{o}$ and $x_{2}-x_{1}=c\left(P_{o}-P_{t}\right)$, as illustrated in the sketch. From this it follows that $C=\frac{x_{2}-x_{1}}{t_{2}-t_{1}}=$ $\frac{P_{o}-P_{t}}{P_{o}} c$. 

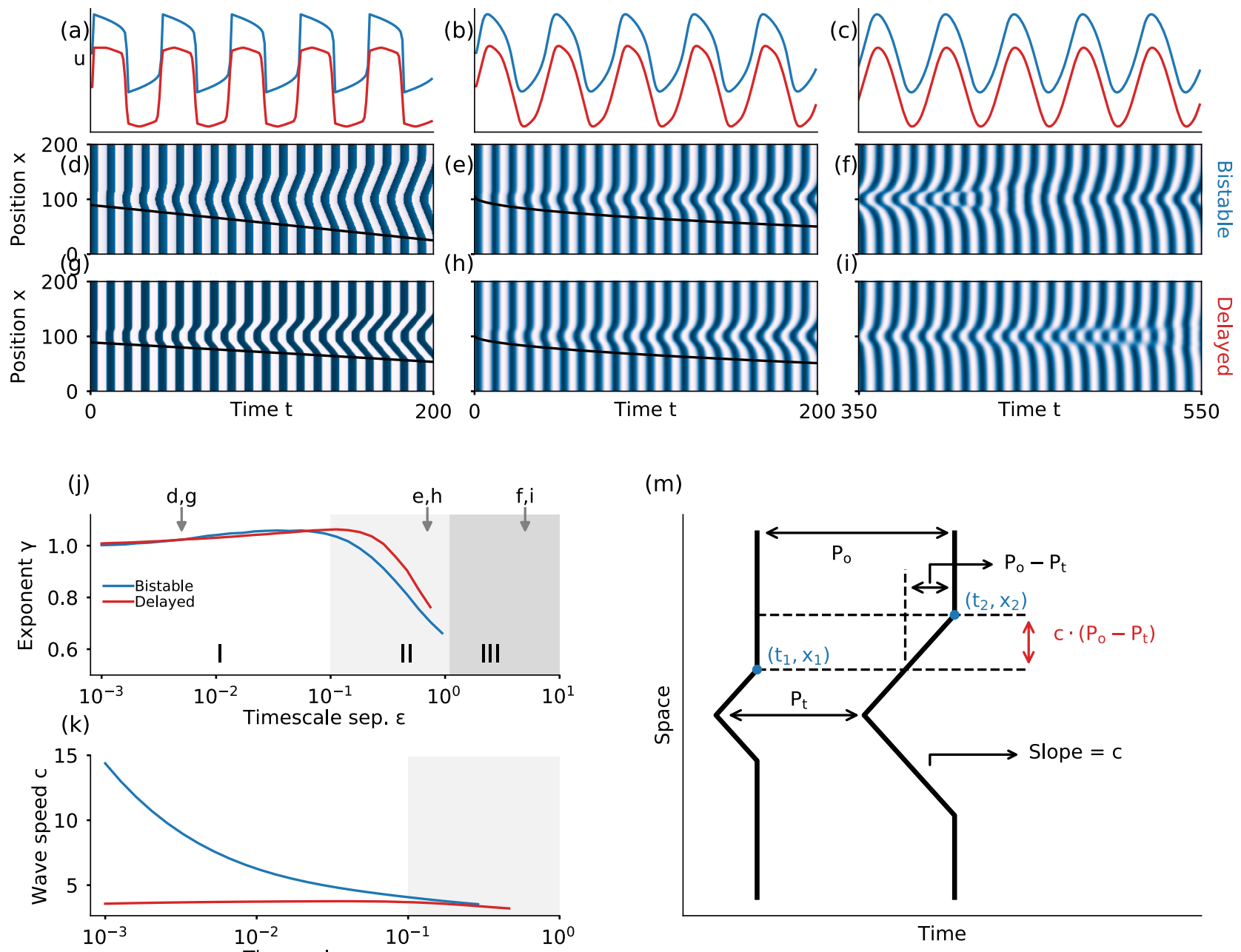

(I)

Timescale sep. $\varepsilon$

(n)
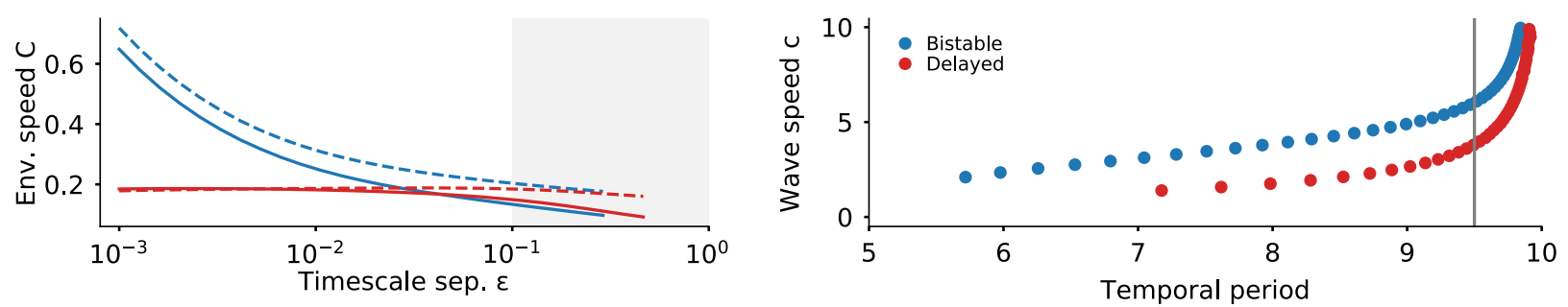

FIG. 5. Wave propagation, wave speed, and envelope speed as functions of timescale separation $\varepsilon$. (a)-(c) Time series of the $u$ variable for $\varepsilon=0.005,0.7$, and 5 for the bistable (blue) and delayed (red) oscillator. The blue lines (bistable) are shifted upwards to better distinguish the shapes. In reality the curves overlap. (d)-(f) Space-time plots for the bistable system. (g)-(i) Space-time plots for the delayed system. For both the bistable and the delayed system we have on the left: $\varepsilon=0.005$, linear wave propagation; middle: $\varepsilon=0.7$, sublinear outward spreading of the pacemaker influence; right: $\varepsilon=5$, phase slips. (j) Exponent of the envelope spread as a function of $\varepsilon$. Three regions are indicated: linear (or faster) spread (I), sublinear spreading (II), and phase slips (III). The arrows indicate the values of $\varepsilon$ used in panels (a)-(i). (k) Wave speed as a function of $\varepsilon$, computed where $\gamma>0.9$. (1) Envelope speed as a function of $\varepsilon$. The dashed lines indicate the approximation $C=\frac{P_{o}-P_{i}}{P_{o}} c$. Other parameters used: $D_{u}=D_{v}=1, S=20, P_{o}=10$, and $P_{i}=9.5 .(\mathrm{m})$ Geometric relation between wave speed, envelope speed, $P_{o}$, and the effective period of the pacemaker $P_{t}$. (n) Dispersion relation for wave trains in the oscillatory medium without inhomogeneity, for $\varepsilon=0.01$. The curves were obtained by simulating the system on a domain of length $\lambda$ until a wave train of a single wavelength was obtained and by computing its speed and temporal period. The system was rescaled such that the period of a uniform oscillation was $P_{o}=10$.

Note, however, that $P_{t}$ here is the effective period of the pacemaker region, taking into account the influence from outside as well. It is not equal to $P_{i}$, the pacemaker's intrinsic frequency, which is a parameter of the model. Due to diffusion, the period of the pacemaker region shifts upward, such that $P_{i}<P_{t}<P_{o}$. This affects the wave speed, since higher periods correspond to higher wave speeds, as can be seen from the dispersion relation for these systems [Fig. 5(n)]. However, 
this mainly plays a role when the size of the pacemaker is smaller, as we discuss in Sec. IVD. The dashed lines in Fig. 5 show the envelope speed if we assume that $P_{t}=P_{i}$. The approximation is generally good and is slightly better for low values of $\varepsilon$. This indicates that the effect from the outside medium on the pacemaker is larger for larger values of $\varepsilon$.

On a side note, a formula for the envelope speed already appears in Hagan's paper [36, Eq. (4.21)]. There, both the envelope speed and the wave speed are derived analytically through a phase-reduction method. The formula we derived above is implicit in the equations in Hagan's paper, but to the best of our knowledge the geometric derivation has not appeared in the literature.

\section{Similar results hold for both a bistable and a delayed cell cycle model}

To verify that our results are not specific to the system based on the Van der Pol oscillator, we perform the same analysis for a bistable model and a time-delayed model that describe cell cycle oscillations in early Xenopus laevis frog embryos. This biological system has been an important example of nonlinear dynamics, both spatial and temporal, generated by biochemical mechanisms. The biochemical underpinnings of these cell cycle oscillations are well characterized. Moreover, in extracts made of these frog eggs, waves of mitosis have been observed [12], and recently the importance of pacemakers in these systems has been shown as well [26,27].

Different mathematical models for this system exist, each taking into account a number of experimental observations. Experiments have shown that in this system both bistability $[48,49]$ and time delay [50,51] play a role. The oscillation is built around periodic activation and inactivation of a protein called cyclin-dependent kinase 1 (Cdk1), which becomes active when bound to a cyclin B subunit. Activated Cdk1 brings about its own decline through activation of a protein complex called the anaphase promoting complex/cyclosome (APC/C), which targets cyclin B for destruction. This negative feedback loop is time delayed. Moreover, Cdk1 is involved in different positive feedback loops, which creates a bistable response as function of cyclin B activity.

We perform the same analysis as in the previous section and numerically compute the wave speed as function of a timescale separation parameter. In the bistable model, there is a natural timescale separation present when using the default, experimentally obtained parameter set. We introduce the additional parameter $\varepsilon$ to modify this inherent timescale separation. For the bistable system, we use the model by Yang and Ferrell [51], and for the delayed model we use a variant of a simple model we studied before [47]. Note that, unlike the Van der Pol variants we study in the rest of the paper, the two cell cycle models here cannot be directly compared. The timescale separation parameter $\varepsilon$ has a different meaning in each model and applies to different variables. Both models, however, are limit cases of a single, general, three-equation model. The details of the equations and parameter values can be found in Appendix C.

The simulations with the cell cycle models confirm what we saw for the Van der Pol system (see Fig. 6): in the system

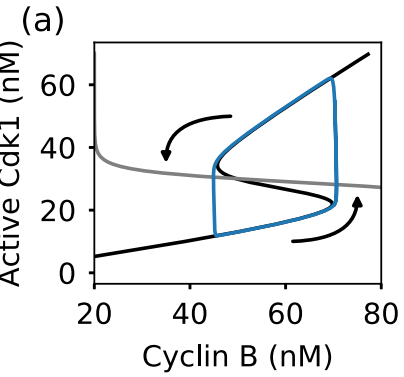

(b)
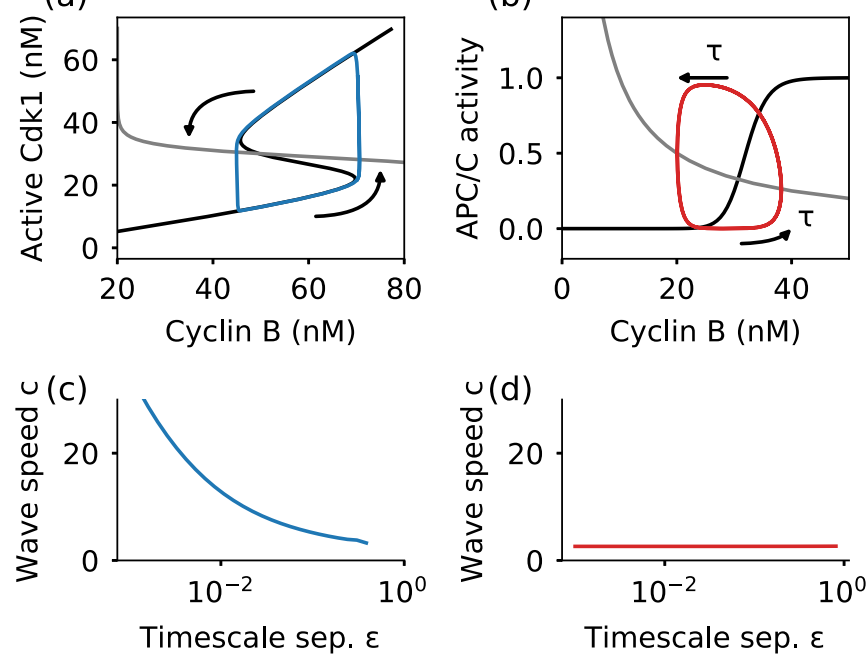

FIG. 6. In two different cell cycle models, timescale separation influences wave speed only when bistability is present. (a) Phase plane representation of the cell cycle oscillation in the bistable model. The system oscillates by repeatedly moving up and down along the branches of a bistable response curve. Black and gray lines are the nullclines of the system. (b) The oscillation in a plane for the delayed cell cycle model. The intersection of the black and gray lines is the fixed point of the system, which is unstable due to time delay. (c) Wave speed as function of $\varepsilon$ for the bistable cell cycle model. (d) Wave speed as function of $\varepsilon$ for the delayed cell cycle model.

based on bistability, the timescale separation determines the speed of the outgoing waves. In the delayed model, there is almost no effect of timescale separation on the wave speed.

Note that in real systems, such as the cell cycle, both time delay and bistability probably play a role in generating the oscillations. Preliminary results on both the Van der Pol-like model as the cell cycle model indicate that, if we add a time delay to a bistable model, the curve representing wave speed as function of $\varepsilon$ changes only little, unless the time delay is large. We leave a more in-depth study of systems in which bistability and time delay are mixed for future work.

\section{Influence of size and period of the pacemaker}

Besides the parameters of the underlying oscillating system, there are two properties of the pacemaker itself that have an important influence on wave behavior: its size and its period [Figs. 7(a)-7(d)]. While larger pacemakers promote linear wave spreading [Fig. 7(e)], they emit waves that are slower [Fig. $7(\mathrm{~g})]$. This is likely due to a mechanism that affects the effective period, which we briefly discussed before. Diffusion shifts the period of the pacemaker region upward to a period $P_{t}$ which lies between $P_{i}$ and $P_{o}$. This effect is stronger for smaller pacemakers, which is intuitive: since they are smaller, they undergo a relatively larger effect from the surroundings, causing their period to shift to values closer to the period of the surrounding medium. Waves with a higher temporal period move faster due to the dispersion relation for wave trains in these systems [Fig. 5(n)]. Therefore smaller pacemakers with their higher period emit faster waves. Perhaps counterintuitively, whereas the wave speed decreases 


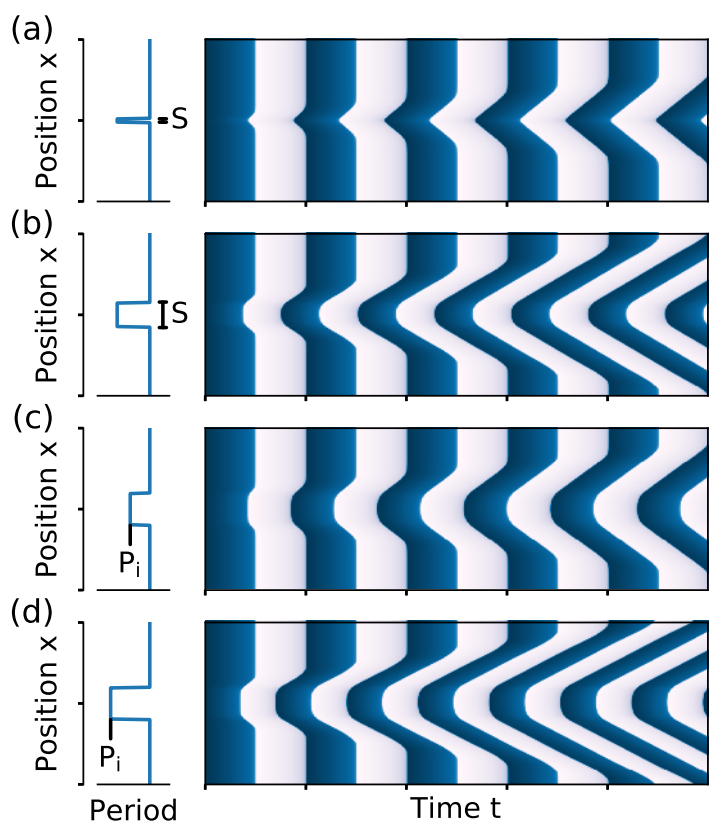

(e)
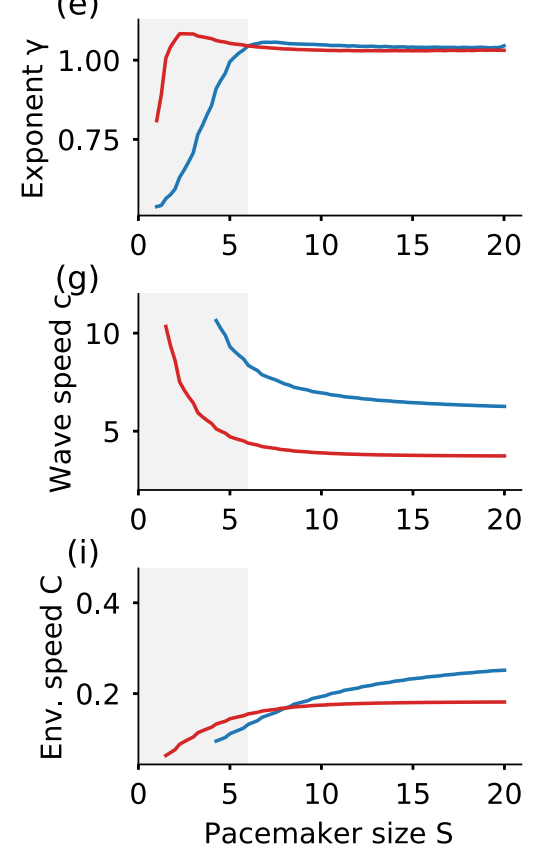

(f)

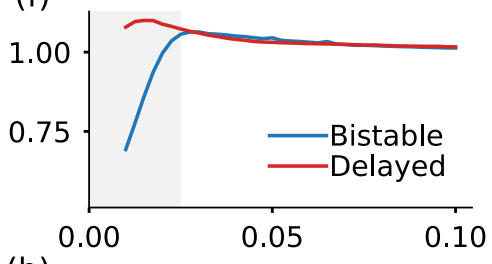

(h)
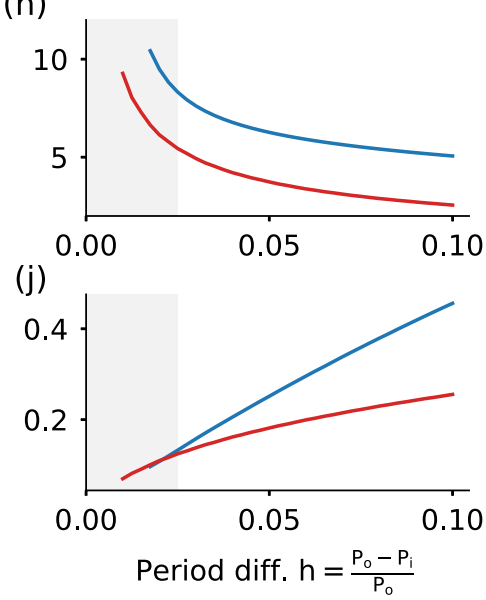

FIG. 7. The size and period of the pacemaker influence the outgoing waves. (a), (b) Space-time plot for waves sent out by a small (a) and a large (b) pacemaker. Waves from the larger pacemaker have a higher envelope speed, but a lower wave speed. (c), (d) Space-time plot for waves sent out by a pacemaker with low (c) and high (d) period difference with the surrounding medium. A larger period difference corresponds to a higher envelope speed and a lower wave speed. (e), (f) Envelope exponent $\gamma$ as a function of the pacemaker size $S$ (e) and the relative difference of the period of the pacemaker and the surrounding medium $h=\left(P_{o}-P_{i}\right) / P_{o}(\mathrm{f}) .(\mathrm{g}),(\mathrm{h})$ Wave speed $c$ as a function of pacemaker size $(\mathrm{g})$ and period difference (h). (i), (j) Envelope speed $C$ as a function of pacemaker size (i) and period difference (j). Parameters: $\varepsilon=0.01, P_{o}=10$, and $D_{u}=D_{v}=1$. $\left(P_{o}-P_{i}\right) / P_{o}=0.05$ in panels (e), (g), and (i). $S=20$ in panels (f), (h), and (j).

with size, the speed of the envelope increases [Fig. 7(i)]. This follows from Eq. (4): whereas $c$ is smaller for a large pacemaker, it maintains a higher difference with the outside medium such that the term $P_{o}-P_{t}$ is larger. This can compensate for a decreasing wave speed $c$. Note that both wave speed and envelope speed saturate as the size becomes large. In this limit, the period of the pacemaker is not shifted due to influence from outside and $P_{t} \rightarrow P_{i}$.

Next, we fix the pacemaker size and the period of the outside medium $P_{o}$, but vary $P_{i}$ : the intrinsic pacemaker period. Here, a similar picture emerges. Define $h=\frac{P_{o}-P_{i}}{P_{o}}$, the relative difference in period between the pacemaker and the medium. A larger value of $h$ ensures that waves spread linearly [Fig. 7(f)], but leads to lower speeds [Fig. 7(h)]. Again, whereas the wave speed decreases, the envelope speed increases [Fig. 7(j)]. Note that when the difference in periods is too large, phase slipping can occur [similar to Figs. 5(f) and 5(i)]. The results on size and frequency hold for both the bistable and the delayed system.

\section{E. Influence of diffusion strength}

The other purely spatial aspect which influences wave propagation is the strength of diffusion. Both types of oscillator differ when it comes to the influence of the diffusion constants $D_{u}$ and $D_{v}$ (Fig. 8). In the bistable system, the speed is mainly determined by the diffusion strength of the fast variable, $D_{u}$ [Figs. 8(a) and 8(c)]. A large value of $D_{v}$ destroys the linear propagation $(\gamma<1)$. In the delayed system, both $D_{u}$ and $D_{v}$ affect the speed of the wave and whether it propagates linearly [Figs. 8(b) and 8(d)]. Typically it is assumed that the wave speed scales as $\sqrt{D}$. For pacemaker-generated waves this is only strictly true in the bistable model with $D_{v}=0$ [Fig. 8(e)]. In the other cases the speed actually increases faster than expected from a $\sqrt{D}$ scaling [Figs. 8(e) and 8(f)]. This is likely again caused by the change in effective period $P_{t}$ : larger diffusion means more influence of the outside medium on the pacemaker. This leads to an increased effective period $P_{t}$ [Figs. 8(g) and 8(h)] and a higher speed, following the dispersion relation [Fig. 5(n)]. Interestingly, our results show that this mechanism is much weaker in the bistable model when only diffusion in the fast $u$ variable is present, indicating that the effective period of the pacemaker $P_{t}$ is mainly influenced by diffusion of the slow variable $v$. Finally, we note that the $D_{v}=0$ case corresponds to typical models of neuronal systems and action potentials, in which the scaling $c \sim \sqrt{D}$ therefore holds.

\section{CONCLUSIONS}

Robust biochemical oscillators, which are essential for the survival and development of many organisms, are often built on interaction motifs which generate bistability and/or time delays. In this paper, we have shown that oscillators built on these mechanisms behave differently when coupled in space. Whereas both a bistable and a delayed oscillator can generate outgoing target patterns, bistable systems generate faster waves provided that the system shows sufficient 

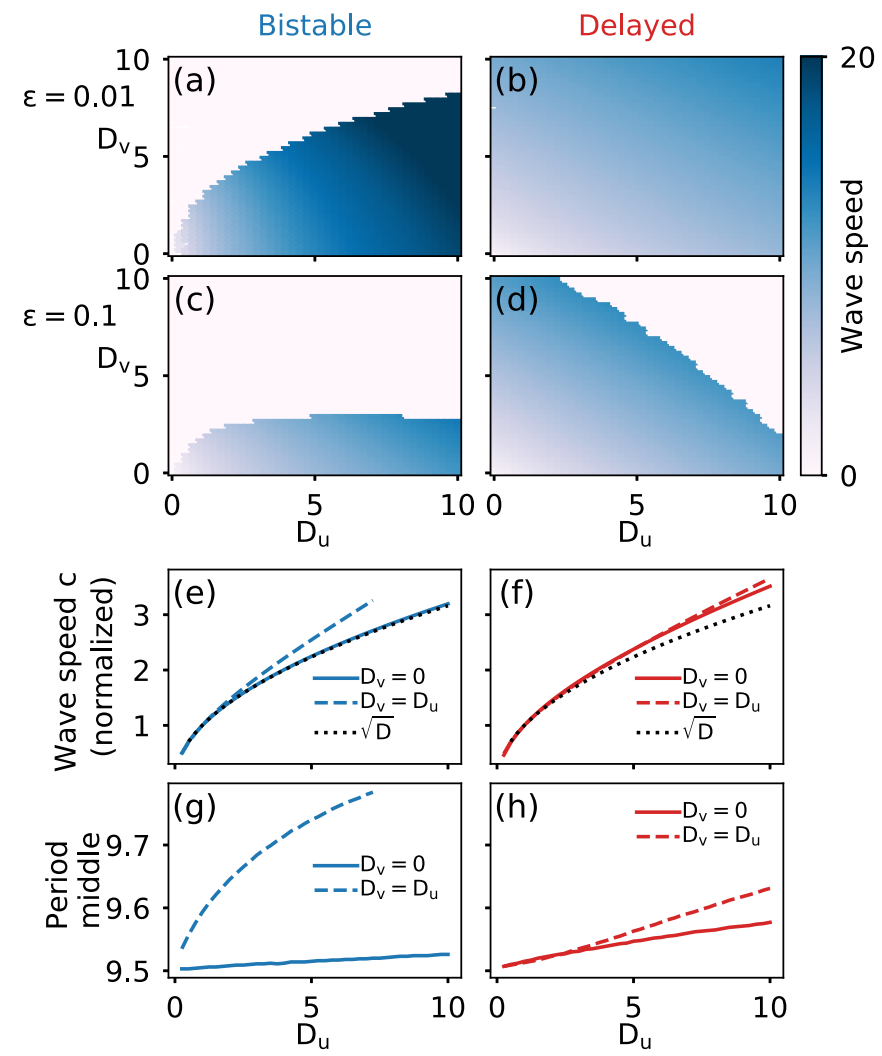

FIG. 8. Influence of the diffusion strength of the two variables on the wave speed. (a)-(d) Wave speed $c$ as a function of $D_{u}$ and $D_{v}$ for the bistable system (a), (c) and the delayed system (b), (d). Two values of the timescale separation are used: $\varepsilon=0.01$ (a), (b) and $\varepsilon=0.1$ (c), (d). In the white region $\gamma<0.9$, no clear, linearly propagating waves are found. (e), (f) Wave speed $c$ as a function of $D_{u}$ for the bistable system (e) and the delayed system (f) for $D_{v}=0$ and $D_{u}=D_{v}(\varepsilon=0.01)$. Values are normalized to their value at $D_{u}=1$. (g), (h) Similar to panels (e) and (f), but now showing the effective period of the pacemaker region as a function of $D_{u}$.

timescale separation. The bistable system is characterized by two regimes. For large timescale separation (small $\varepsilon$ ), the wave speed is markedly higher than for small timescale separation. In the latter regime, there is little difference between bistable and delayed oscillators. The higher wave speed in the bistable system is most likely due to the fact that for large timescale separation, waves in this system are governed by the propagation of fronts between the two stable branches. This observation is also the starting point of the singular perturbation approach to traveling waves [32] and is applicable to excitable media as well as oscillatory media.

For high $\varepsilon$, the oscillations are nearly harmonic and the wave dynamics are no longer governed by front propagation. In this situation, the dynamics are better described through a phase-reduction approach such as the one typically used in theoretical studies of pacemakers which we discussed in the Introduction. In a follow-up paper, we intend to discuss these different approximation methods and their application to pacemaker-generated waves.

We have found that pacemakers need not send out a wave which permeates linearly into the medium: large dif- fusion, small timescale separation, and/or small pacemakers can lead to sublinear spreading, in our terminology corresponding to $\gamma<1$. Another interesting aspect of our study is the explicit relation between wave speed and envelope speed. Perhaps contrary to expectations, the size and the frequency of the pacemaker influence wave speed and envelope spreading speed in different ways. A large pacemaker, or one with a larger frequency difference, is usually considered to be a strong pacemaker. Strong pacemakers do take over the medium faster, due to the increased envelope speed, but the waves they send out are slower. Due to the generality of these results, they will likely also apply to the classical examples of target patterns and pacemakers, namely the chemical oscillators such as the Belousov-Zhabotinsky reaction.

The distinction between envelope speed and wave speed is also important in situations where multiple pacemakers are present in a single medium. When this is the case, they compete with each other until one takes over the whole medium. We recently investigated such systems of multiple pacemakers [52] and showed that both size and frequency matter in the determination of which pacemaker dominates the whole medium.

Understanding which factors contribute to the speed and range of pacemaker-generated waves is important as these types of waves have increasingly been shown to play a crucial role in coordinating biological processes over large distances $[1,11,16]$. It is therefore reasonable to expect that evolution has somehow optimized these systems to be able to transfer information as fast as possible, maybe bound to other constraints on the interactions between proteins and the spatial structure of cells. In our study we have given a general overview of the factors that determine the wave speed of a pacemaker-generated wave, for different types of oscillators and different parameter regimes. We expect that such a general overview is an important step to better understand which mechanisms are the most biologically relevant.

\section{ACKNOWLEDGMENTS}

This work was supported by the Research Foundation Flanders (FWO) with individual support to J.R. and project support to L.G. (Grant No. GOA5317N) and the KU Leuven Research Fund (Grant No. C14/18/084). The computational resources and services used in this work were provided by the VSC (Flemish Supercomputer Center) and the Flemish Government—department EWI.

\section{APPENDIX A: ABSORPTION OF THE PARAMETERS $d$ AND $b$}

The set of equations for the bistable system is as follows:

$$
\begin{gathered}
u_{t}=\varepsilon^{-1}\left(v-\frac{1}{4} d u\left(u^{2}-b\right)\right)+D_{u} u_{x x}, \\
v_{t}=a-u+D_{v} v_{x x} .
\end{gathered}
$$

By the change of variables

$\tilde{u}=\frac{2}{\sqrt{b}} u, \quad \tilde{v}=\frac{8}{b \sqrt{b} d} v, \quad s=\frac{4}{d b} t, \quad y=\frac{2}{\sqrt{d b}} x$, 
this system can be written as

$$
\begin{gathered}
\tilde{u}_{s}=\tilde{\varepsilon}^{-1}\left(\tilde{v}-\frac{1}{4} \tilde{u}\left(\tilde{u}^{2}-4\right)\right)+D_{u} \tilde{u}_{y y}, \\
\tilde{v}_{s}=\tilde{a}-\tilde{u}+D_{v} \tilde{v}_{y y},
\end{gathered}
$$

where

$$
\tilde{\varepsilon}=\frac{16}{(d b)^{2}} \varepsilon, \quad \tilde{a}=\frac{2}{\sqrt{b}} a .
$$

Here, the parameters $b$ and $d$, which determined the shape of the cubic nullcline, have been absorbed in $\varepsilon$ and $a$.

\section{APPENDIX B: DETAILS OF THE NUMERICAL SCHEME}

Simulations of the set of PDEs were done in PYTHON using the NUMPY and SCIPY modules. We tested multiple methods and settled on a pseudospectral method for the bistable system and an implicit method for the delayed system. These provided the best compromise between accuracy, stability, and simplicity. Especially for the fast-moving waves (low $\varepsilon$ in the bistable model), the pseudospectral method gives the most accurate results.

\section{Pseudospectral method}

Let $\mathcal{F}$ denote the spatial Fourier tranform. We integrate the system

$$
X_{t}=F(X)+D X_{x x}
$$

as follows. Let $t_{i}=t_{0}+i d t$ be the time points and $x_{j}, j=$ $0, \ldots, N-1$ be the spatial grid points.

$$
\left(\begin{array}{ccccc}
1+2 \alpha & -\alpha & 0 & \cdots & -\alpha \\
-\alpha & 1+2 \alpha & -\alpha & \cdots & 0 \\
0 & -\alpha & 1+2 \alpha & \cdots & 0 \\
\vdots & \ddots & \ddots & \ddots & \vdots \\
-\alpha & \cdots & 0 & -\alpha & 1+2 \alpha
\end{array}\right)\left(\begin{array}{c}
X_{i+1,1} \\
X_{i+1,2} \\
\vdots \\
X_{i+1, N-1} \\
X_{i+1, N}
\end{array}\right)=\left(\begin{array}{c}
X_{i, 1}+\operatorname{dtF}\left(X_{i, 1}, X_{i-n, 1}\right) \\
X_{i, 2}+\operatorname{dtF}\left(X_{i, 2}, X_{i-n, 2}\right) \\
\vdots \\
X_{i, N-1}+d t F\left(X_{i, N-1}, X_{i-n, N-1}\right) \\
X_{i, N}+d t F\left(X_{i, N}, X_{i-n, N}\right)
\end{array}\right)
$$

(i) Perform one Euler step of the reaction term over half a time step:

$$
X_{i+\frac{1}{2}, j}=X_{i, j}+\frac{d t}{2} F\left(X_{i, j}\right) .
$$

(ii) Perform the diffusion part in Fourier space over a full time step:

$$
\begin{aligned}
& \hat{X}_{i+\frac{1}{2}, k}=\mathcal{F}\left(X_{i+\frac{1}{2}, j}\right), \\
& \hat{\tilde{X}}_{i+\frac{1}{2}, k}=\hat{X}_{i+\frac{1}{2}, k}+d t D\left(-k^{2}\right) \hat{X}_{i+\frac{1}{2}, k}, \\
& \tilde{X}_{i+\frac{1}{2}, j}=\mathcal{F}^{-1}\left(\hat{\tilde{X}}_{i+\frac{1}{2}, k}\right) .
\end{aligned}
$$

(iii) Perform another Euler step over $d t / 2$ :

$$
X_{i+1, j}=\tilde{X}_{i+\frac{1}{2}, j}+\frac{d t}{2} F\left(\tilde{X}_{i+\frac{1}{2}, j}\right) .
$$

We use SCIPY's FFT algorithms to quickly compute the transforms.

\section{Implicit method}

For systems with delay, we use an implicit method. We discretize the equation $X_{t}=F[X(t), X(t-\tau)]+D X_{x x}$ as follows (with $\tau=n d t$ ):

$$
\begin{aligned}
\frac{X_{i+1, j}-X_{i, j}}{d t}= & F\left(X_{i, j}, X_{i-n, j}\right)+\frac{D}{d x^{2}}\left(X_{i+1, j-1}\right. \\
& \left.-2 X_{i+1, j}+X_{i+1, j+1}\right) .
\end{aligned}
$$

Set $\alpha=\frac{D d t}{d x}$. In matrix form the equation becomes
This means we first perform the reaction step (with delay) with a forward Euler step, and then the diffusion step with a backward Euler step. We solve the resulting matrix equation at each timestep using SCIPY's sparse matrix solver.

Our standard simulation time is $T=1000$ with $d t=0.001$ and $N=4096$ spatial grid points. For the simulations whose results are shown in Figs. 5(j)-5(1) we used $d t=0.0001$ and for Fig. 8 we used $N=1024$. This takes a lot of computation time, so we perform simulations on a high-performance computing cluster provided by VSC (Flemish Supercomputer Center).

\section{Rescaling of time}

In order to compare the different wave speeds for different parameters, we rescale all the systems we study such that they have a period $P_{o}$. If the original nonspatial oscillator (bistable or delayed) is described by

$$
X^{\prime}=F[X(t), X(t-\tau)],
$$

where in our case $X=(u, v)^{T}$, for each value of the parameters, we first numerically compute the period of the limit cycle, call it $P$. The rescaled system

$$
X^{\prime}=\frac{P}{P_{o}} F\left[X(t), X\left(t-\tau \frac{P_{o}}{P}\right)\right]
$$

then has period $P_{o}$. In order to introduce the inhomogeneity, we rescale time again in the middle part of the region such that the period there is $P_{i}$. Note that the shape of the limit cycle is exactly the same in the middle and the outside regions, only the speed by which it is traversed is different.

\section{APPENDIX C: EQUATIONS OF THE CELL CYCLE MODELS}

\section{Bistable model}

The bistable cell cycle model is taken from Yang and Ferrell [51]. The equations 
TABLE II. Parameter values for the model of cyclin-Cdk1 interaction by Yang and Ferrell [51].

\begin{tabular}{ccc}
\hline \hline Symbol & Meaning & Value \\
\hline$k_{s}$ & Cyclin production rate & $1 \mathrm{nM} / \mathrm{min}$ \\
$a_{\mathrm{deg}}$ & Basal APC/C activity & 0.01 \\
$b_{\mathrm{deg}}$ & Maximal APC/C activity & 0.04 \\
$\mathrm{EC} 50_{\mathrm{deg}}$ & Threshold for APC/C activation & $32 \mathrm{nM}$ \\
$n_{\mathrm{deg}}$ & Hill exponent for APC/C activation & 17 \\
$a_{\mathrm{Cdc25}}$ & Basal Cdc25 activity & 0.16 \\
$b_{\mathrm{Cdc25}}$ & Maximal Cdc25 activity & 0.8 \\
$\mathrm{EC} 50_{\mathrm{Cdc} 25}$ & Threshold for Cdc25 activation & $35 \mathrm{nM}$ \\
$n_{\mathrm{Cdc25}}$ & Hill exponent for Cdc25 activation & 11 \\
$a_{\mathrm{Wee} 1}$ & Basal Wee1 activity & 0.08 \\
$b_{\mathrm{Wee} 1}$ & Maximal Wee1 activity & 0.4 \\
$\mathrm{EC} 50_{\mathrm{Wee} 1}$ & Threshold for Wee1 activation & $30 \mathrm{nM}$ \\
$n_{\mathrm{Wee} 1}$ & Hill exponent for Wee1 activation & 3.5 \\
\hline \hline
\end{tabular}

are

$$
\begin{aligned}
& u^{\prime}=k_{s}-a(u) u+\varepsilon^{-1}[c(u)(v-u)-w(u) u], \\
& v^{\prime}=k_{s}-a(u) v
\end{aligned}
$$

where

$$
\begin{gathered}
a(u)=a_{\mathrm{deg}}+b_{\mathrm{deg}} \frac{u^{n_{\mathrm{deg}}}}{\mathrm{EC} 50_{\mathrm{deg}}^{n_{\mathrm{deg}}}+u^{n_{\mathrm{deg}}}}, \\
c(u)=a_{\mathrm{Cdc} 25}+b_{\mathrm{Cdc} 25} \frac{u^{n_{\mathrm{Cdc} 25}}}{\mathrm{EC} 50_{\mathrm{Cdc} 25}^{n_{\mathrm{Cd} 25}}+u^{n_{\mathrm{Cdc} 25}}}, \\
w(u)=a_{\mathrm{Wee} 1}+b_{\mathrm{Wee} 1} \frac{\mathrm{EC} 50_{\mathrm{Wee} 1}^{n_{\mathrm{Wee} 1}}}{\mathrm{EC} 50_{\mathrm{Wee} 1}^{n_{\mathrm{Wee} 1}}+u^{n_{\mathrm{Wee} 1}}} .
\end{gathered}
$$

Here, $v$ is the slow variable which denotes the total amount of Cdk1-cyclin B complexes in the cell. The fast variable $u$ stands for active Cdk1-cyclin B complexes. Cyclin B is produced at a constant rate $k_{s}$ and assumed to bind instantly to $\mathrm{Cdk} 1$ to create the complex. It is targeted for degradation by APC/C, a protein whose activity depends on active Cdk1-cyclin B through the function $a(u)$. The production and degradation are considered to be slow. The activation and inactivation of the Cdk1-cyclin B complex happen on a faster timescale. The complex can activate itself, through an intermediate protein called $\mathrm{Cdc} 25$, and inactivate itself through Wee1. Both of these proteins depend on the active Cdk1cyclin B through Hill functions, respectively, $c(u)$ and $w(u)$. We have introduced the parameter $\varepsilon$ in this model to modulate the timescale separation between (in)activation kinetics and production/degradation. Note that if $\varepsilon=1$, the fast reactions are already approximately 10 times faster than the slow ones. In Fig. 6(c) in the main text, we have therefore divided the
TABLE III. Parameters for the delayed cell cycle model.

\begin{tabular}{ccc}
\hline \hline Symbol & Meaning & Value \\
\hline$k_{s}$ & Cyclin B production rate & $1 \mathrm{nM} / \mathrm{min}$ \\
$b_{\mathrm{deg}}$ & Maximal cyclin B degradation rate & $0.11 / \mathrm{min}$ \\
$K$ & Threshold for APC/C activation & $32 \mathrm{nM}$ \\
$n$ & Hill exponent for APC/C activation & 17 \\
$\tau$ & Time delay for APC/C activation & $10 \mathrm{~min}$ \\
\hline \hline
\end{tabular}

actual value of $\varepsilon$ by 10 for plotting and comparing with the other oscillator. The values of the other parameters used can be found in Table II.

\section{Delayed model}

The delayed cell cycle model is given by the equations

$$
\begin{aligned}
u^{\prime} & =\varepsilon^{-1}\left(\frac{v(t-\tau)^{n}}{K^{n}+v(t-\tau)^{n}}-u\right), \\
v^{\prime} & =k_{s}-b_{\mathrm{deg}} u v .
\end{aligned}
$$

Here, $v$ is Cdk1-cyclin B activity. In this model, the positive feedback loops through Wee1 and Cdc25 are not present (see Tsai et al. [53] for a discussion of the strength of the feedback loops), and there is no bistability. This effectively means that all Cdk1-cyclin B complexes are active. There is no variable to denote the total amount of complexes. On the other hand, we now include a variable for APC/C activity, $u$. APC/C is activated quickly by $\mathrm{Cdk} 1-$ cyclin $\mathrm{B}$ after a time delay. For $\varepsilon \rightarrow 0, \mathrm{APC} / \mathrm{C}$ activation becomes instant and the above system of equations reduces to

$$
v^{\prime}=k_{s}-b_{\operatorname{deg}} \frac{v(t-\tau)^{n}}{K^{n}+v(t-\tau)^{n}} v .
$$

We have studied this model in detail in a previous paper [47]. The parameters we use for simulating the cell cycle model (C5) are given in Table III.

\section{Both cell cycle models are limit cases of one three-equation model}

The bistable cell cycle model and the delayed cell cycle model can both be seen as limits of one three-equation model:

$$
\begin{aligned}
& u^{\prime}=k_{s}-y u+\delta^{-1}[c(u)(v-u)-w(u) u], \\
& v^{\prime}=k_{s}-y v, \\
& y^{\prime}=\eta^{-1}\{a[u(t-\tau)]-y\} .
\end{aligned}
$$

Here $u$ is active Cdk1-cyclin $\mathrm{B}, v$ is total Cdk1-cyclin $\mathrm{B}$, and $y$ stands for APC/C. For $\eta \rightarrow 0$ and $\tau=0$, this reduces to the bistable cell cycle model. For $\delta \rightarrow \infty$, this model reduces to the delayed cell cycle model.
[1] C. Beta and K. Kruse, Annu. Rev. Condens. Matter Phys. 8, 239 (2017).

[2] A. T. Winfree, J. Theor. Biol. 16, 15 (1967).

[3] Y. Kuramoto, Chemical Oscillations, Waves, and Turbulence, Springer Series in Synergetics (Springer-Verlag, Berlin, 1984).
[4] R. Thomas, in Numerical Methods in the Study of Critical Phenomena, Springer Series in Synergetics, edited by J. Della Dora, J. Demongeot, and B. Lacolle (Springer, Berlin, 1981), pp. 180-193.

[5] D. Thieffry and M. Kaufman, J. Theor. Biol. 474, 42 (2019). 
[6] T. Y.-C. Tsai, Y. S. Choi, W. Ma, J. R. Pomerening, C. Tang, and J. E. Ferrell, Jr., Science 321, 126 (2008).

[7] B. Novák and J. J. Tyson, Nat. Rev. Mol. Cell Biol. 9, 981 (2008).

[8] J. E. Ferrell, Jr., T. Y.-C. Tsai, and Q. Yang, Cell 144, 874 (2011).

[9] T. Erneux, Applied Delay Differential Equations, Surveys and Tutorials in the Applied Mathematical Sciences (SpringerVerlag, New York, 2009).

[10] M. C. Cross and P. C. Hohenberg, Rev. Mod. Phys. 65, 851 (1993).

[11] L. Gelens, G. Anderson, and J. E. Ferrell, Jr., Mol. Biol. Cell 25, 3486 (2014).

[12] J. B. Chang and J. E. Ferrell, Jr., Nature (London) 500, 603 (2013).

[13] V. E. Deneke, A. Melbinger, M. Vergassola, and S. Di Talia, Dev. Cell 38, 399 (2016).

[14] J. Allard and A. Mogilner, Curr. Opin. Cell Biol.25, 107 (2013).

[15] N. Inagaki and H. Katsuno, Trends Cell Biol. 27, 515 (2017).

[16] V. Deneke and S. Di Talia, J. Cell Biol. 217, 1193 (2018).

[17] G. B. Ermentrout and D. H. Terman, Mathematical Foundations of Neuroscience, Interdisciplinary Applied Mathematics, Vol. 35 (Springer, Berlin, 2010).

[18] S. Alonso, M. Bär, and B. Echebarria, Rep. Prog. Phys. 79, 096601 (2016).

[19] A. F. Taylor, Prog. React. Kinet. Mech. 27, 247 (2002).

[20] S. Jakubith, H. H. Rotermund, W. Engel, A. von Oertzen, and G. Ertl, Phys. Rev. Lett. 65, 3013 (1990).

[21] J. Wolff, M. Stich, C. Beta, and H. H. Rotermund, J. Phys. Chem. B 108, 14282 (2004).

[22] C. Punckt, M. Stich, C. Beta, and H. H. Rotermund, Phys. Rev. E 77, 046222 (2008).

[23] B. Till, H. G. Othmer, and C. J. Weijer, Interface Focus 6, 20160047 (2016).

[24] J. Schütze, T. Mair, M. J. B. Hauser, M. Falcke, and J. Wolf, Biophys. J. 100, 809 (2011).

[25] L. Muller, F. Chavane, J. Reynolds, and T. J. Sejnowski, Nat. Rev. Neurosci. 19, 255 (2018).

[26] F. E. Nolet, A. Vandervelde, A. Vanderbeke, L. Pineros, J. B. Chang, and L. Gelens, eLife 9, e52868 (2020).

[27] O. Afanzar, G. K. Buss, T. Stearns, and J. Ferrell, Jr., bioRxiv:2020.06.16.153437.

[28] C. van Oss, A. V. Panfilov, P. Hogeweg, F. Siegert, and C. J. Weijer, J. Theor. Biol. 181, 203 (1996).

[29] P. M. Wood and J. Ross, J. Chem. Phys. 82, 1924 (1985).

[30] B. J. Caldwell, M. L. Trew, G. B. Sands, D. A. Hooks, I. J. LeGrice, and B. H. Smaill, Circ.: Arrhythmia Electrophysiol. 2, 433 (2009).
[31] A. L. Hodgkin and A. F. Huxley, J. Physiol. 117, 500 (1952).

[32] J. J. Tyson and J. P. Keener, Physica D (Amsterdam, Neth.) 32, 327 (1988).

[33] W. van Saarloos, Phys. Rep. 386, 29 (2003).

[34] J. Keener and J. Sneyd, Mathematical Physiology: I. Cellular Physiology, 2nd ed., Interdisciplinary Applied Mathematics (Springer-Verlag, New York, 2009).

[35] J. Murray, Mathematical Biology, 3rd ed., Interdisciplinary Applied Mathematics, Vol. 17 (Springer, Berlin, 2002).

[36] P. S. Hagan, Adv. Appl. Math. 2, 400 (1981).

[37] J. C. Neu, SIAM J. Appl. Math. 36, 7 (1979).

[38] N. Kopell, Adv. Appl. Math. 2, 389 (1981).

[39] V. García-Morales and K. Krischer, Contemp. Phys. 53, 79 (2012).

[40] M. Stich and A. S. Mikhailov, Physica D (Amsterdam, Neth.) 215, 38 (2006).

[41] H. Mahara, T. Saito, Y. Amagishi, H. Nagashima, and T. Yamaguchi, J. Phys. Soc. Jpn. 69, 3552 (2000).

[42] M. Stich and A. S. Mikhailov, Z. Phys. Chem. 216, 521 (2002).

[43] B. Van der Pol, London, Edinburgh, Dublin Philos. Mag. J. Sci. 2, 978 (1926).

[44] R. FitzHugh, Biophys. J. 1, 445 (1961).

[45] J. Nagumo, S. Arimoto, and S. Yoshizawa, Proc. Inst. Radio Eng. 50, 2061 (1962).

[46] B. C. Goodwin, Adv. Enzyme Regul. 3, 425 (1965).

[47] J. Rombouts, A. Vandervelde, and L. Gelens, PLoS One 13, e0194769 (2018).

[48] J. R. Pomerening, E. D. Sontag, and J. E. Ferrell, Jr., Nat. Cell Biol. 5, 346 (2003).

[49] W. Sha, J. Moore, K. Chen, A. D. Lassaletta, C.-S. Yi, J. J. Tyson, and J. C. Sible, Proc. Natl. Acad. Sci. USA 100, 975 (2003).

[50] M.-A. Félix, J.-C. Labbé, M. Dorée, T. Hunt, and E. Karsenti, Nature (London) 346, 379 (1990).

[51] Q. Yang and J. E. Ferrell, Jr., Nat. Cell Biol. 15, 519 (2013).

[52] F. E. Nolet, J. Rombouts, and L. Gelens, Chaos 30, 053139 (2020).

[53] T. Y.-C. Tsai, J. A. Theriot, and J. Ferrell, Jr., PLoS Biol. 12, e1001788 (2014).

Correction: A minor error in Eq. (C4) has been fixed.

Second Correction: Errors in notation and text in the paragraph below Eq. (4) have been fixed. Values given in the last paragraph of Sec. 2 in Appendix B have been fixed, along with corresponding text. 